\title{
Immediate Endoscopic Management of an Intramural Hematoma Developed during Colonoscopy
}

\author{
Chang-II Kwon, Duck Hwan Kim and Sung Pyo Hong \\ Digestive Disease Center, CHA Bundang Medical Center, CHA University, Seongnam, Korea
}

An intramural hematoma of the gastrointestinal tract could be precipitated by an endoscopic examination, especially in patients being administered anticoagulation therapy or those with concomitant hematological diseases. ${ }^{1}$ Although an intramural hematoma can be primarily associated with a biopsy or therapeutic procedure, occurrence of a spontaneous intramural hematoma during a diagnostic colonoscopy has been reported in a few cases. ${ }^{2-4}$ Intramural hematomas are known to cause partial or complete bowel obstruction, ${ }^{5}$ and surgical treatment should be considered in patients with evidence of intestinal necrosis, peritonitis, or a deteriorating general condition despite conservative management. ${ }^{6,7}$ Early detection and treatment of a hematoma, can prevent bowel obstruction and obviate the need for surgery. Our report describes early endoscopic management of an intramural hematoma, which occurred during a diagnostic colonoscopy.

A 78-year-old man underwent colonoscopy during scheduled follow-up for the management of ulcerative colitis. He had not received anticoagulation therapy, and laboratory tests revealed a normal platelet count and coagulation profile. During colonoscopic examination, a large elongated intramural hematoma with a normal mucosa was seen to develop in the sigmoid colon (Fig. 1A). We concluded the intramural hematoma might have occurred as a consequence of a continu-

Received: March 6, 2017 Revised: May 27, 2017

Accepted: July 10, 2017

Correspondence: Sung Pyo Hong

Digestive Disease Center, CHA Bundang Medical Center, CHA University, 59 Yatap-ro, Bundang-gu, Seongnam13496, Korea

Tel: +82-31-780-5641, Fax: +82-31-780-5219, E-mail: sphong@cha.ac.kr

(cc) This is an Open Access article distributed under the terms of the Creative Commons Attribution Non-Commercial License (http://creativecommons.org/ licenses/by-nc/3.0) which permits unrestricted non-commercial use, distribution, and reproduction in any medium, provided the original work is properly cited. ous suctioning process employed during the procedure due to poor bowel preparation. Even after 10 minutes of observation, we found that the hematoma continued to grow in size due to active submucosal bleeding. We placed three endoscopic clips (HX-610-090L; Olympus, Tokyo, Japan) at the suspected sites of active bleeding (Fig. 1B, arrows), which led to cessation of growth of the hematoma. To determine reliably, a Dual knife (Olympus, Tokyo, Japan) was applied to excise the hematoma at its center and thereby evacuate it (Fig. 1C). An electrosurgical unit (VIO 300D; ERBE Elektromedizin, Tübingen, Germany) with an Endocut I setting (effect 3, duration 3, interval 3) was used as a power source. After excision, we noted immediate active bleeding (Fig. 1D) followed by flattening of the hematoma without secondary bleeding (Fig. 2A). Follow-up examination after 3 hours of observation showed no re-growth of the hematoma, and no further bleeding was documented (Fig. 2B). The patient was discharged without admission or need for further treatment.

\section{Conflicts of Interest}

The authors have no financial conflicts of interest.

\section{REFERENCES}

1. Liu Y, Yang S, Tong Q. Spontaneous intramural hematoma of colon. Clin Gastroenterol Hepatol 2012;10:e38.

2. Gallo D, Tebrock C, Rivera D. Intramural cecal hematoma: an unusual complication of colonoscopy. Gastrointest Endosc 2003;57:254-257.

3. Jongwutiwes $U$, Shaukat $A$, Pocha $C$. Image of the month. Intramural cecal hematoma: a rare complication after colonoscopy. Clin Gastroenterol Hepatol 2009;7:A32.

4. Katsurahara M, Horiki N, Kitade T, et al. Acute colonic intramural hematoma: a rare complication of colonoscopy. Endoscopy 2014;46 Suppl 1 UCTN:E180-E181.

5. Hughes CE 3rd, Conn J Jr, Sherman JO. Intramural hematoma of the gastrointestinal tract. Am J Surg 1977;133:276-279. 


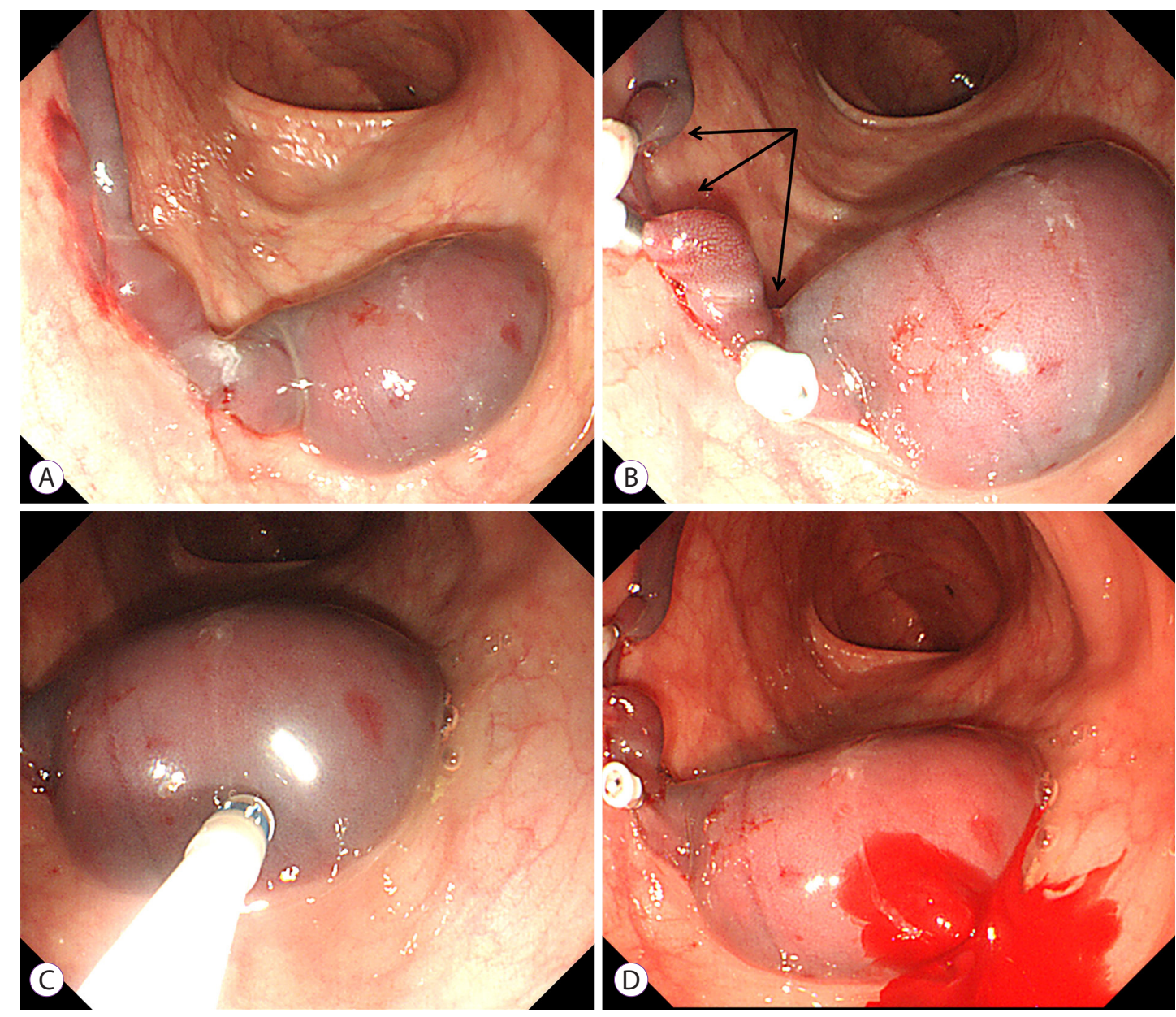

Fig. 1. Colonoscopic images showing an intramural hematoma. (A) An intramural hematoma observed in the sigmoid colon. (B) Rapid increase in the size of the intramural hematoma, and placement of three endoscopic clips at the suspected sites of active bleeding (arrows). (C) Application of Dual knife (Olympus, Tokyo, Japan) to excise and evacuate the hematoma. (D) Active bleeding noted from the hematoma.

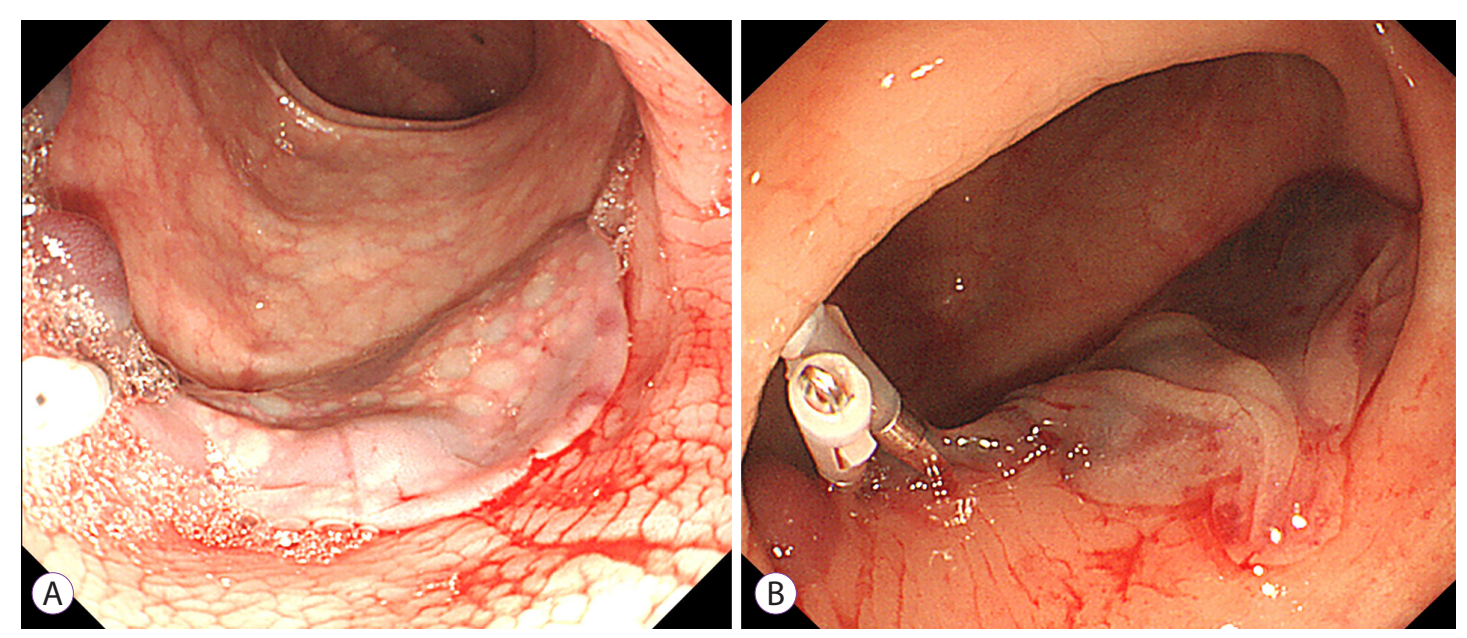

Fig. 2. Follow-up colonoscopic images after endoscopic treatment. (A) Flattened hematoma without secondary bleeding. (B) No evidence of further bleeding after 3 hours of observation.

6. Kwon K, Cheung DY, Seo Y, et al. Supportive management resolved a colonic intramural hematoma in an anticoagulant user. Intern Med 2014;53:1505-1509.
7. Lobo L, Koudki R, Prasad Hl K, Shetty B. Colon obstruction due to an anticoagulant induced intramural haematoma; a rare case report. J Clin Diagn Res 2013;7:739-741. 